\title{
COUNTABLE BOREL EQUIVALENCE RELATIONS
}

\author{
SCOTT SCHNEIDER AND SIMON THOMAS
}

Introduction. These notes are an account of a day-long lecture workshop presented by Simon Thomas of Rutgers University at the University of Ohio at Athens on November 17, 2007, as part of the Appalachian Set Theory series. The workshop served as an intensive introduction to the emerging theory of countable Borel equivalence relations. These notes have been compiled from the lecture slides by Scott Schneider, an attendee of the workshop.

\section{First Session}

1.1. Standard Borel Spaces. A topological space is said to be Polish if it admits a complete, separable metric. If $\mathcal{B}$ is a $\sigma$-algebra of subsets of a given set $X$, then the pair $(X, \mathcal{B})$ is called a standard Borel space if there exists a Polish topology $\mathcal{T}$ on $X$ that generates $\mathcal{B}$ as its Borel $\sigma$-algebra. For example, each of the sets $\mathbb{R},[0,1], \mathbb{N}^{\mathbb{N}}$, and $2^{\mathbb{N}}=\mathcal{P}(\mathbb{N})$ is Polish in its natural topology, and so may be viewed, equipped with its corresponding Borel structure, as a standard Borel space.

The abstraction involved in passing from a topology to its associated Borel structure is analagous to that of passing from a metric to its induced topology. Just as distinct metrics on a space may induce the same topology, distinct topologies may very well generate the same Borel $\sigma$-algebra. In a standard Borel space, then, one "remembers" only the Borel sets, and forgets which of them were open; it is natural therefore to imagine that any of them might have been, and indeed this is the case:

Theorem 1.1.1. Let $(X, \mathcal{T})$ be a Polish space and $Y \subseteq X$ any Borel subset. Then there exists a Polish topology $\mathcal{T}_{Y} \supseteq \mathcal{T}$ such that $\mathcal{B}\left(\mathcal{T}_{Y}\right)=\mathcal{B}(\mathcal{T})$ and $Y$ is clopen in $\left(X, \mathcal{T}_{Y}\right)$.

It follows that if $(X, \mathcal{B})$ is a standard Borel space with $Y \in \mathcal{B}$, then $(T, \mathcal{B} \uparrow Y)$ is also a standard Borel space. In fact, so much structual information is "forgotten" in passing from a Polish space to its Borel structure that we obtain the following theorem of Kuratowski [18].

Theorem 1.1.2. There exists a unique uncountable standard Borel space up to isomorphism. 
A remarkably wide range of naturally occurring classes of mathematical objects may be viewed as standard Borel spaces. In fact, experience has shown that practically anything one is able to "write down" or describe explicitly in a way that does not make us of the Axiom of Choice can be treated in the context of some suitably defined standard Borel space.

1.2. Borel Equivalence Relations. It turns out that many classification problems from diverse areas of mathematics may be viewed as equivalence relations on suitably defined standard Borel spaces. For example, consider the problem of classifying all countable graphs up to graph isomorphism. Letting $\mathcal{C}$ be the set of graphs of the form $\Gamma=\langle\mathbb{N}, E\rangle$ and identifying each graph $\Gamma \in \mathcal{C}$ with its edge relation $E \in 2^{\mathbb{N}^{2}}$, one easily checks that $\mathcal{C}$ is a Borel subset of $2^{\mathbb{N}^{2}}$, and hence is itself a standard Borel space. Moreover, the isomorphism relation on $\mathcal{C}$ is simply the orbit equivalence relation arising from the natural action of $\operatorname{Sym}(\mathbb{N})$ on $\mathcal{C}$. More generally, if $\sigma$ is a sentence of $\mathcal{L}_{\omega_{1}, \omega}$, then

$$
\operatorname{Mod}(\sigma)=\{\mathcal{M}=\langle\mathbb{N}, \cdots\rangle \mid \mathcal{M} \models \sigma\}
$$

is a standard Borel space, and the isomorphism relation on $\operatorname{Mod}(\sigma)$ is the orbit equivalence relation generated by the $\operatorname{Sym}(\mathbb{N})$ action. However, while this orbit equivalence relation is always analytic, it is not in general Borel; for instance, the graph isomorphism relation on $\mathcal{C}$ is not Borel. On the other hand, the restriction of graph isomorphism to the standard Borel space of countable locally finite graphs is Borel, and in general the isomorphism relation on a standard Borel space of countable structures which are "finitely generated" in some broad sense will be Borel. With these examples in mind we make the following definitions.

Definition 1.2.1. Let $X$ be a standard Borel space. Then a Borel equivalence relation on $X$ is an equivalence relation $E \subseteq X^{2}$ which is a Borel subset of $X^{2}$.

Definition 1.2.2. Let $G$ be a Polish group. Then a standard Borel $G$-space is a standard Borel space $X$ equipped with a Borel action $(g, x) \mapsto g \cdot x$. The corresponding $G$-orbit equivalence relation is denoted by $E_{G}^{X}$.

We observe that if $G$ is a countable group and $X$ is a standard Borel $G$-space, then $E_{G}^{X}$ is a Borel equivalence relation. As further examples, we consider the standard Borel space $R\left(\mathbb{Q}^{n}\right)$ of torsion-free abelian groups of rank $n$ and the Polish space $\mathcal{G}$ of finitely generated groups. 


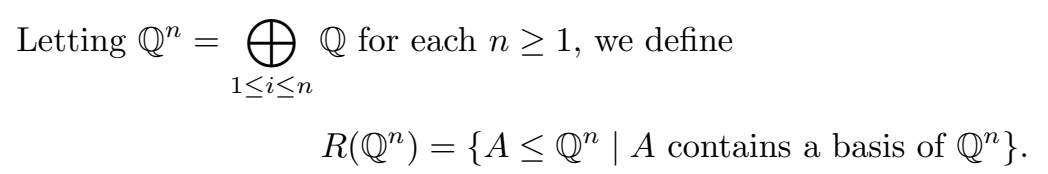

Then for $A, B \in R\left(\mathbb{Q}^{n}\right)$, we have that

$$
A \cong B \quad \text { iff } \quad \text { there exists } \varphi \in G L_{n}(\mathbb{Q}) \text { such that } \varphi(A)=B
$$

and hence the isomorphism relation on $R\left(\mathbb{Q}^{n}\right)$ is the Borel equivalence relation arising from the natural $G L_{n}\left(\mathbb{Q}^{n}\right)$ action on $R\left(\mathbb{Q}^{n}\right)$.

For the Polish space $\mathcal{G}$ of finitely generated groups, we first define, for each $m \in \mathbb{N}, \mathcal{G}_{m}$ to be the compact space of normal subgroups of the free group $\mathbb{F}_{m}$ on the $m$ generators $\left\{x_{1}, \ldots, x_{m}\right\}$. Since each $m$-generator group can be realized as a quotient $\mathbb{F}_{m} / N$ for some $N \in \mathcal{G}_{m}$, we can regard $\mathcal{G}_{m}$ as the space of $m$-generator groups. We then have natural embeddings

$$
\mathcal{G}_{1} \hookrightarrow \mathcal{G}_{2} \hookrightarrow \cdots \hookrightarrow \mathcal{G}_{m} \hookrightarrow \cdots
$$

and can regard

$$
\mathcal{G}=\bigcup_{m \geq 1} \mathcal{G}_{m}
$$

as the space of finitely generated groups.

By a theorem of Tietze, if $N, M \in \mathcal{G}_{m}$, then $\mathbb{F}_{m} / N \cong \mathbb{F}_{m} / M$ if and only if there exists $\pi \in \operatorname{Aut}\left(\mathbb{F}_{2 m}\right)$ such that $\pi(N)=M$. It follows that the isomorphism relation $\cong$ on the space $\mathcal{G}$ of finitely generated groups is the orbit equivalence relation arising form the homeomorphic action of the countable group $\operatorname{Aut}_{f}\left(\mathbb{F}_{\infty}\right)$ of finitary automorphisms of the free group $\mathbb{F}_{\infty}$ on $\left\{x_{1}, x_{2}, \cdots, x_{m}, \cdots\right\}$.

1.3. Borel Reducibility. Evidently various naturally occurring classification problems may be viewed as Borel equivalence relations on standard Borel spaces. In particular, the complexity of the problem of finding complete invariants for such classification problems can be gauged to some extent by the "structural complexity" of the associated Borel equivalence relations. Here the crucial notion of comparison is that of a Borel reduction.

Definition 1.3.1. If $E$ and $F$ are Borel equivalence relations on the standard Borel spaces $X$, $Y$ respectively, then we say that $E$ is Borel reducible to $F$, and write $E \leq_{B} F$, if there exists a Borel map $f: X \rightarrow Y$ such that $x E y \leftrightarrow f(x) F f(y)$. Such a map is called a Borel reduction from $E$ to $F$. If $f: X \rightarrow Y$ satisfies the weaker condition that $x E y \rightarrow f(x) F f(y)$, then $f$ is 
called a Borel homomorphism from $E$ to $F$. We say that $E$ and $F$ are Borel bireducible, and write $E \sim_{B} F$, if both $E \leq_{B} F$ and $F \leq_{B} E$; and we write $E<_{B} F$ if $E \leq_{B} F$ but $F \not_{B} E$.

If $E$ and $F$ are Borel equivalence relations, then we interpret $E \leq_{B} F$ to mean that the classification problem associated with $E$ is at most as complicated as that associated with $F$, in the sense that an assignment of complete invariants for $F$ would, via composition with the Borel reduction from $E$ to $F$, yield one for $E$ as well. Additionally we observe that if $f: E \leq_{B} F$, then the induced map $\tilde{f}: X / E \rightarrow Y / F$ is an embedding of quotient spaces, the existence of which is sometimes interpreted as saying that $X / E$ has "Borel cardinality" less than or equal to that of $Y / F$.

This notion of Borel reducibility imposes a partial (pre)-order on the collection of Borel equivalence relations, and much of the work currently taking place in the theory of Borel equivalence relations concerns determining the structure of this partial ordering. For a long time, many questions about this structure remained open, and it was notoriously difficult to obtain non-reducibility results. More recently, however, some progress has been made in establishing benchmarks within the $\leq_{B}$-hierarchy. For instance, an important breakthrough occurred in 2000 when Adams and Kechris [2] proved that the partial ordering of Borel sets under inclusion embeds into the $\leq_{B}$ ordering on the subclass of countable Borel equivalence relations, which we shall define shortly.

As a first step towards describing the $\leq_{B}$-hierarchy, we introduce the so-called smooth and hyperfinite Borel equivalence relations. Writing $\mathrm{id}_{\mathbb{R}}$ for the identity relation on $\mathbb{R}$, we begin with the following theorem of Silver [24]:

Theorem 1.3.2 (Silver). If $E$ is a Borel equivalence relation with uncountably many classes, then $i d_{\mathbb{R}} \leq_{B} E$.

Hence $\operatorname{id}_{\mathbb{R}}$ - and any Borel equivalence relation bireducible with it - is a $\leq{ }_{B}$-minimal element in the partial ordering of Borel equivalence relations with uncountably many classes. We call such relations smooth.

Definition 1.3.3. The Borel equivalence relation $E$ is smooth iff $E \leq_{B}$ id $d_{X}$ for some (equivalently every) uncountable standard Borel space $X$.

As an example, the isomorphism problem on the space of countable divisible abelian groups is smooth. Furthermore, if $\equiv$ is the equivalence relation defined on the space $\mathcal{G}$ of finitely 
generated groups by $G \equiv H$ iff Th $G=$ Th $H$, then $\equiv$ is smooth. For an example of a non-smooth Borel equivalence relation, we turn to the following:

Definition 1.3.4. $E_{0}$ is the Borel equivalence relation defined on $2^{\mathbb{N}}$ by $x E_{0} y$ iff $x(n)=y(n)$ for all but finitely many $n$.

To see that $E_{0}$ is not smooth, suppose $f: 2^{\mathbb{N}} \rightarrow[0,1]$ is a Borel reduction from $E_{0}$ to $\operatorname{id}_{[0,1]}$, and let $\mu$ be the usual product probability measure on $2^{\mathbb{N}}$. Then $f^{-1}\left(\left[0, \frac{1}{2}\right]\right)$ and $f^{-1}\left(\left[\frac{1}{2}, 1\right]\right)$ are Borel tail events, so by Kolmogorov's zero-one law, either $\mu\left(f^{-1}\left(\left[0, \frac{1}{2}\right]\right)\right)=1$ or $\mu\left(f^{-1}\left(\left[\frac{1}{2}, 1\right]\right)\right)=1$. Continuing to cut intervals in half in this manner, we obtain that $f$ is $\mu$-a.e. constant, a contradiction.

1.4. Countable Borel Equivalence Relations. An important subclass of Borel equivalence relations consists of those with countable sections.

Definition 1.4.1. A Borel equivalence relation on a standard Borel space is called countable if each of its equivalence classes is countable.

The importance of this subclass stems in large part from the fact that each such equivalence relation can be realized as the orbit equivalence relation of a Borel action of a countable group. Of course, if $G$ is a countable group and $X$ a standard Borel $G$-space, then the corresponding orbit equivalence relation $E_{G}^{X}$ is a countable Borel equivalence relation. But by a remarkable result of Feldman and Moore [8], the converse is also true:

Theorem 1.4.2 (Feldman-Moore). If $E$ is a countable Borel equivalence relation on the standard Borel space $X$, then there exists a countable group $G$ and a Borel action of $G$ on $X$ such that $E=E_{G}^{X}$.

Sketch of Proof. (See [26, 5.8.13]). Let $E$ be a countable Borel equivalence relation on the standard Borel space $X$. Since $E \subseteq X^{2}$ has countable sections, the Lusin-Novikov Uniformization Theorem $[17,18.10]$ implies that we can write $E$ as a countable union of graphs of injective partial Borel functions, $f_{n}: \operatorname{dom} f_{n} \rightarrow X$. Each $f_{n}$ is easily modified into a Borel bijection $g_{n}: X \rightarrow X$ with the same "orbits." But then $E$ is simply the orbit equivalence relation arising from the resulting Borel action of the group $G=\left\langle g_{n} \mid n \in \mathbb{N}\right\rangle$.

Unfortunately, the countable group and its action given by the Feldman-Moore theorem are by no means canonical. For example, let us define the Turing equivalence relation $\equiv_{T}$ on 
$\mathcal{P}(\mathbb{N})$ by

$$
A \equiv_{T} B \quad \text { iff } \quad A \leq_{T} B \wedge B \leq_{T} A
$$

where $\leq_{T}$ denotes Turing reducibility. Then $\equiv_{T}$ is clearly a countable Borel equivalence relation, and hence by Feldman-Moore it must arise as the orbit equivalence relation induced by a Borel action of some countable group $G$ on $\mathcal{P}(\mathbb{N})$. However, the proof of the theorem gives us no information about $G$ or this action, and so it is reasonable to ask:

Vague Question 1.4.3. Can $\equiv_{T}$ be realized as the orbit equivalence relation of a " $n i c e "$ Borel action of some countable group?

We have seen that there is a $\leq_{B}$-minimal Borel equivalence relation on an uncountable standard Borel space. While there is no maximal relation in the general setting, the subclass of countable Borel equivalence relations does indeed admit a universal element, by a result of Dougherty, Jackson, and Kechris [7].

Definition 1.4.4. A countable Borel equivalence relation $E$ is universal iff $F \leq_{B} E$ for every countable Borel equivalence relation $F$.

This universal countable Borel equivalence relation can be realized as follows. Let $\mathbb{F}_{\omega}$ be the free group on infinitely many generators, and define a Borel action of $\mathbb{F}_{\omega}$ on

$$
\left(2^{\mathbb{N}}\right)^{\mathbb{F}_{\omega}}=\left\{p \mid p: \mathbb{F}_{\omega} \rightarrow 2^{\mathbb{N}}\right\}
$$

by setting

$$
(g \cdot p)(h)=p\left(g^{-1} h\right), \quad p \in\left(2^{\mathbb{N}}\right)^{\mathbb{F} \omega} .
$$

Let $E_{\omega}$ be the resulting orbit equivalence relation.

Claim 1.4.5. $E_{\omega}$ is a universal countable Borel equivalence relation.

Proof. Let $X$ be a standard Borel space and let $E$ be any countable Borel equivalence relation on $X$. Since every countable group is a homomorphic image of $\mathbb{F}_{\omega}$, by Feldman-Moore it follows that $E$ is the orbit equivalence relation of a Borel action of $\mathbb{F}_{\omega}$. Let $\left\{U_{i}\right\}_{i \in \mathbb{N}}$ be a sequence of Borel subsets of $X$ which separates points and define $f: X \rightarrow\left(2^{\mathbb{N}}\right)^{\mathbb{F}_{\omega}}$ by $x \mapsto f_{x}$, where

$$
f_{x}(h)(i)=1 \quad \text { iff } \quad x \in h\left(U_{i}\right) .
$$


Then $f$ is injective and

$$
\begin{array}{rll}
\left(g \cdot f_{x}\right)(h)(i)=1 & \text { iff } & f_{x}\left(g^{-1} h\right)(i)=1 \\
& \text { iff } & x \in g^{-1} h\left(U_{i}\right) \\
& \text { iff } & g \cdot x \in h\left(U_{i}\right) \\
& \text { iff } & f_{g \cdot x}(h)(i)=1
\end{array}
$$

Another universal countable Borel equivalence relation is the orbit equivalence relation $E_{\infty}$ arising from the translation action of the free group $\mathbb{F}_{2}$ on its powerset. Of course, any two universal countable Borel equivalence relations are Borel bireducible, so we often speak of "the" (up to $\sim_{B}$ ) universal countable Borel equivalence relation.

We have now seen that within the class of countable Borel equivalence relations, there exist a $\leq_{B}$-least and a $\leq_{B}$-greatest element, up to $\sim_{B}$, with realizations id $\mathbb{R}$ and $E_{\infty}$, respectively. It turns out that the minimal id ${ }_{\mathbb{R}}$ has an immediate $\leq_{B}$-successor [11]:

Theorem 1.4.6 (The Glimm-Effros Dichotomy). If $E$ is nonsmooth Borel, then $E_{0} \leq_{B} E$.

We call a Borel equivalence relation $E$ hyperfinite if it can be written as the increasing union $E=\cup_{n} F_{n}$ of a sequence of Borel equivalence relations with finite classes. It is easily shown that $E_{0}$ is hyperfinite, and in fact it is the case that every nonsmooth hyperfinte countable Borel equivalence relation is Borel bireducible with $E_{0}$. Furthermore, by a result of Dougherty, Jackson, and Kechris [7], if $E$ is a countable Borel equivalence relation, then $E$ can be realized as the orbit equivalence relation of a Borel $\mathbb{Z}$-action if and only if $E \leq_{B} E_{0}$. Finally, by the Adams-Kechris [2] result mentioned above, we know that there exist $2^{\aleph_{0}}$ distinct countable Borel equivalence relations up to Borel bireducibility. Combining these basic facts gives the following picture of the universe of countable Borel equivalence relations. 


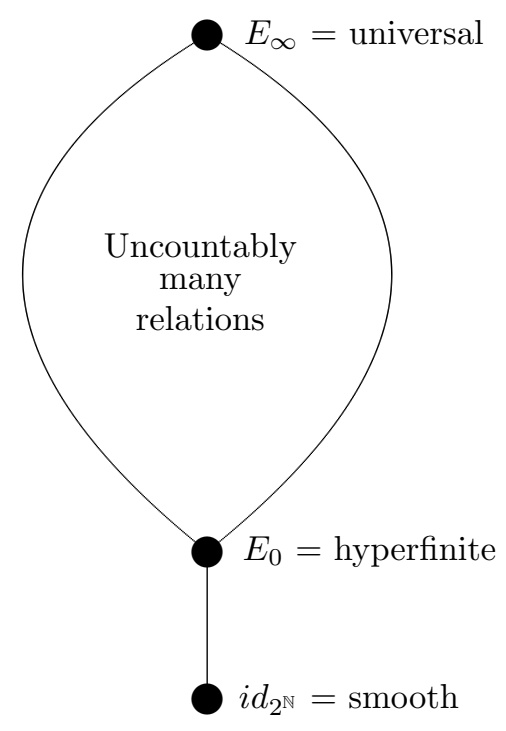

Given this picture, one might ask where a particular countable Borel equivalence relation lies relative to the known benchmarks. In the following section we shall consider this question for the Turing equivalence relation, $\equiv_{T}$. Here Martin has conjectured that $\equiv_{T}$ is not universal, while Kechris has conjectured that it is. However, despite some progress, which we discuss below, the problem remains open.

1.5. Turing Equivalence and The Martin Conjectures. We first define the set of Turing degrees to be the collection

$$
\mathcal{D}=\left\{a=[A]_{\equiv_{T}} \mid A \in \mathcal{P}(\mathbb{N})\right\}
$$

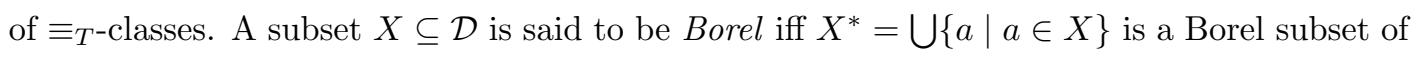
$\mathcal{P}(\mathbb{N})$. It is well known that if $E$ is a Borel equivalence relation on a standard Borel space $X$, then the quotient space $X / E$ is standard Borel if and only if $E$ is smooth. Since $\equiv_{T}$ is not smooth, it follows that $\mathcal{D}$ is not a standard Borel space.

For $a, b \in \mathcal{D}$, we define $a \leq b$ iff $A \leq_{T} B$ for each $A \in a$ and $B \in b$; and for each $a \in \mathcal{D}$, we define the corresponding cone $C_{a}=\{b \in \mathcal{D} \mid a \leq b\}$. Of course, each $C_{a}$ is a Borel subset of $\mathcal{D}$.

Theorem 1.5.1 (Martin). If $X \subseteq \mathcal{D}$ is Borel, then for some $a \in \mathcal{D}$, either $C_{a} \subseteq X$ or $C_{a} \subseteq \mathcal{D} \backslash X$. 
Proof. Let $X \subseteq \mathcal{D}$ be Borel and consider the 2-player game $G\left(X^{*}\right)$

$$
a=a(0) a(1) a(2) \cdots \quad \text { where each } a(n) \in 2
$$

such that Player 1 wins iff $a \in X^{*}$. Then $G\left(X^{*}\right)$ is Borel and hence is determined. Suppose that $\varphi: 2^{<\mathbb{N}} \rightarrow 2$ is a winning strategy for Player 1 . We claim that $C_{\varphi} \subseteq X$.

To see this, suppose that $\varphi \leq_{T} x$ and let Player 2 play $x=a(1) a(3) a(5) \cdots$. Then $y=\varphi(x) \in X^{*}$ and $x \equiv_{T} y$. It follows that $x \in X^{*}$.

For later use, note that if $X \subseteq \mathcal{D}$ is Borel, then $X$ contains a cone iff $X$ is cofinal in $\mathcal{D}$.

Similarly, we define a function $f: \mathcal{D} \rightarrow \mathcal{D}$ to be Borel iff there exists a Borel function $\varphi: \mathcal{P}(\mathbb{N}) \rightarrow \mathcal{P}(\mathbb{N})$ such that $f\left([A]_{\equiv_{T}}\right)=[\varphi(A)]_{\equiv_{T}}$. We are now ready to state the following conjecture of Martin, which will implies that $\equiv_{T}$ is not universal.

Conjecture 1.5.2 (Martin). If $f: \mathcal{D} \rightarrow \mathcal{D}$ is Borel, then either $f$ is constant on a cone or else $f(a) \geq a$ on a cone.

While this conjecture remains open, there do exist some partial results of Slaman and Steel [25] that point in its direction:

Theorem 1.5.3 (Slaman-Steel). If $f: \mathcal{D} \rightarrow \mathcal{D}$ is Borel and $f(a)<a$ on a cone, then $f$ is constant on a cone.

Theorem 1.5.4 (Slaman-Steel). If the Borel map $f: \mathcal{D} \rightarrow \mathcal{D}$ is uniformly invariant, then either $f$ is constant on a cone or else $f(a) \geq a$ on a cone.

The definition of a uniformly invariant map can be found in Slaman-Steel [25].

In order to see that the Martin conjecture implies that $\equiv_{T}$ is not universal, note that if $\equiv_{T}$ is universal then $\left(\equiv_{T} \times \equiv_{T}\right) \sim_{B} \equiv_{T}$, whence there exist Borel complete sections $Y \subseteq$ $\mathcal{P}(\mathbb{N}) \times \mathcal{P}(\mathbb{N}), Z \subseteq \mathcal{P}(\mathbb{N})$ and a Borel isomorphism

$$
f:\left\langle Y,\left(\equiv_{T} \times \equiv_{T}\right) \uparrow Y\right\rangle \rightarrow\left\langle Z, \equiv_{T}\lceil Z\rangle .\right.
$$

This isomorphism induces a Borel pairing function $f: \mathcal{D} \times \mathcal{D} \rightarrow \mathcal{D}$. Now fix $d_{0} \neq d_{1} \in \mathcal{D}$ and define the Borel maps $f_{i}: \mathcal{D} \rightarrow \mathcal{D}$ by $f_{i}(a)=f\left(d_{i}, a\right)$. By the Martin Conjecture, $f_{i}(a) \geq a$ on a cone and so ran $f_{i}$ are cofinal Borel subsets of $\mathcal{D}$. Hence each ran $f_{i}$ contains a cone, which is impossible since $\operatorname{ran} f_{0} \cap \operatorname{ran} f_{1}=\emptyset$. 
Letting $\leq_{A}$ denote arithmetic reducibility, we define the arithmetic equivalence relation $\equiv_{A}$ on $\mathcal{P}(\mathbb{N})$ by

$$
B \equiv_{A} C \text { iff } B \leq_{A} C \wedge C \leq_{A} B
$$

It is a theorem of Slaman and Steel that $\equiv_{A}$ is a universal countable Borel equivalence relation. One might take this as evidence that $\equiv_{T}$ is also universal. However, as Slaman has pointed out, an important difference between the two cases is that the arithmetic degrees have less closure with respect to arithmetic equivalences than the Turing degrees do for recursive equivalences.

\section{SECOND SESSION}

\subsection{The Fundamental Question in the Theory of Countable Borel Equivalence}

Relations. We have already seen, by the remarkable result of Feldman and Moore, that every countable Borel equivalence relation on a standard Borel space arises as the orbit equivalence relation of some Borel action of a suitable countable group. We have also seen, however, that this action is not canonically determined, and that it is sometimes difficult to express a given countable Borel equivalence relation as the orbit equivalence relation arising from a "natural" group action. Since many of the techniques currently available for analyzing countable Borel equivalence relations deal with properties of the groups and actions from which they arise, one of the fundamental questions in the theory concerns the extent to which an orbit equivalence relation $E_{G}^{X}$ determines the group $G$ and its action on $X$. Ideally one would hope for the complexity of $E_{G}^{X}$ to reflect the complexity of $G$, so that relations $E_{G}^{X}$ and $E_{H}^{X}$ can be distinguished (in the sense of $\leq_{B}$ ) by distinguishing $G$ from $H$.

Of course, strong hypotheses on a countable group $G$ and its action on a standard Borel space $X$ must be made if there is to be any hope of recovering $G$ and its action from $E_{G}^{X}$, or, even worse, from the Borel complexity of $E_{G}^{X}$ alone. For example, let $G$ be any countable group and consider the Borel action of $G$ on $G \times[0,1]$ defined by $g \cdot(h, r)=(g h, r)$. Then the Borel map $(h, r) \mapsto\left(1_{G}, r\right)$ selects a point in each $G$-orbit, and so the corresponding orbit equivalence relation is smooth. Notice, however, that this action does not admit an invariant probability measure. In fact, we have the following important observation:

Proposition 2.1.1. If $G$ acts freely on $X$ and preserves a probability measure, then $E_{G}^{X}$ is not smooth. 
It turns out that each of these properties is necessary if we are serious about recovering $G$ and its action from $E_{G}^{X}$, as the following two theorems suggest.

Theorem 2.1.2 (Dougherty-Jackson-Kechris [7]). Let $G$ be a countable group and let $X$ be a standard Borel $G$-space. If $X$ does not admit a $G$-invariant probability measure, then for every countable group $H \supset G$, there exists a Borel action of $H$ on $X$ such that $E_{H}^{X}=E_{G}^{X}$.

Theorem 2.1.3. If $E$ is a countable Borel equivalence relation in which every E-class is infinite, then $E$ can be realized as the orbit equivalence relation of a faithful Borel action of uncountably many distinct countable groups.

Definition 2.1.4. A countable Borel equivalence relation in which every E-class is infinite is called aperiodic.

Hence we shall be especially concerned with free, measure-preserving Borel actions of countable groups on standard Borel probability spaces. A natural question, then, is whether we can always hope for this setting:

Question 2.1.5. Let $E$ be a nonsmooth countable Borel equivalence relation. Does there necessarily exist a countable group $G$ with a free measure-preserving Borel action on a standard probability space $(X, \mu)$ such that $E \sim_{B} E_{G}^{X}$ ?

We first observe that half of this question is easily answered: namely, if $E$ is a countable Borel equivalence relation on an uncountable standard Borel space $Y$, then there exists a countable group $G$ and a standard Borel $G$-space $X$ such that $G$ preserves a nonatomic probability measure $\mu$ on $X$, and $E \sim_{B} E_{G}^{X}$. Before considering freeness, we shall need some definitions.

Definition 2.1.6. A Borel action of a countable group $G$ on the standard Borel space $X$ is free iff $g \cdot x \neq x$ for all $1 \neq g \in G$ and $x \in X$. In this case we say that $X$ is a free standard Borel $G$-space.

Definition 2.1.7. The countable Borel equivalence relation $E$ on $X$ is free iff there exists a countable group $G$ with a free Borel action on $X$ such that $E_{G}^{X}=E$.

Definition 2.1.8. The countable Borel equivalence relation $E$ is essentially free iff there exists a free countable Borel equivalence relation $F$ such that $E \sim_{B} F$. 
The obvious question, then, is the following:

Question 2.1.9 (Jackson-Kechris-Louveau [15]). Is every countable Borel equivalence relation essentially free?

2.2. Essentially Free Countable Borel Equivalence Relations. In order to answer Question 2.1.9, it will first be helpful to list some closure properties of essential freeness.

Theorem 2.2.1 (Jackson-Kechris-Louveau [15]). Let E, F be countable Borel equivalence relations on the standard Borel spaces $X, Y$ respectively.

- If $E \leq_{B} F$ and $F$ is essentially free, then so is $E$.

- If $E \subseteq F$ and $F$ is essentially free, then so is $E$.

It follows that every countable Borel equivalence relation is essentially free if and only if $E_{\infty}$ is essentially free.

Theorem 2.2.2 (Thomas 2006, [27]). The class of essentially free countable Borel equivalence relations does not admit a universal element. In particular, $E_{\infty}$ is not essentially free.

Thus, unfortunately, the answer to Question 2.1.5 is no. As a corollary to 2.2.2, we observe that $\equiv_{T}$ is not essentially free; for identifying the free group $\mathbb{F}_{2}$ with a suitably chosen group of recursive permutations of $\mathbb{N}$, we have that $E_{\infty} \subseteq \equiv_{T}$.

This gives us the following map of the universe of nonsmooth countable Borel equivalence relations.

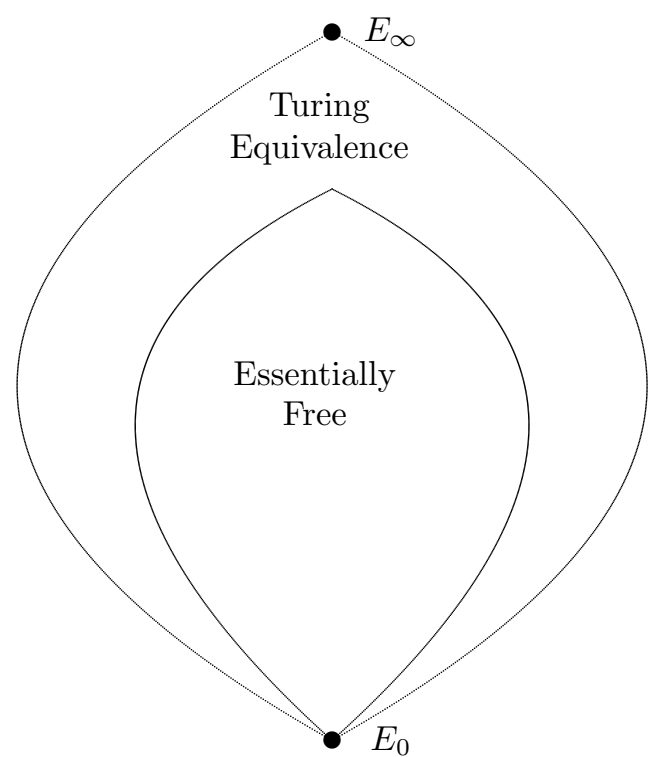


2.3. Bernoulli Actions, Popa Superrigidity, and a Proof of Theorem 2.2.2. In this section we present a proof of Theorem 2.2.2 from an easy consequence of Popa's Superrigidity Theorem. We begin by considering Bernoulli actions.

By a Bernoulli action we mean the shift action of a countably infinite discrete group $G$ on its powerset $\mathcal{P}(G)=2^{G}$. (This is a special case of the notion as it appears in [23]). Under this action the usual product probability measure $\mu$ on $2^{G}$ is $G$-invariant and the free part

$$
\mathcal{P}^{*}(G)=(2)^{G}=\left\{x \in 2^{G} \mid g \cdot x \neq x \text { for all } 1 \neq g \in G\right\}
$$

has $\mu$-measure 1 . We let $E_{G}$ denote the corresponding orbit equivalence relation on $(2)^{G}$, and make the following observation:

Proposition 2.3.1. If $G \leq H$, then $E_{G} \leq_{B} E_{H}$.

Proof. The inclusion map $\mathcal{P}^{*}(G) \hookrightarrow \mathcal{P}^{*}(H)$ is a Borel reduction from $E_{G}$ to $E_{H}$.

Now we just need a few more preliminary definitions before stating the consequence of Popa's theorem we need to prove 2.2.2.

Definition 2.3.2. Let $E$ be a countable Borel equivalence relation on the standard Borel space $X$ with invariant probability measure $\mu$, and let $F$ be a countable Borel equivalence relation on the standard Borel space $Y$. Then the Borel homomorphism $f: X \rightarrow Y$ from $E$ to $F$ is said to be $\mu$-trivial iff there exists a Borel subset $Z \subseteq X$ with $\mu(Z)=1$ such that $f$ maps $Z$ into a single $F$-class.

Definition 2.3.3. If $G$ and $H$ are countable groups, then the homomorphism $\pi: G \rightarrow H$ is $a$ virtual embedding iff $\mid$ ker $\pi \mid<\infty$.

Now we are finally ready to state the consequence of Popa's Cocycle Superrigidity Theorem [23] that we shall use to prove Theorem 2.2.2. We shall discuss Popa's theorem and the proof of this consequence from it at a later point in these notes.

Theorem 2.3.4. Let $G=S L_{3}(\mathbb{Z}) \times S$, where $S$ is any countable group. Let $H$ be any countable group, and let $Y$ be a free standard Borel $H$-space. If there exists a $\mu$-nontrivial Borel homomorphism from $E_{G}$ to $E_{H}^{Y}$, then there exists a virtual embedding $\pi: G \rightarrow H$.

We observe that in particular this conclusion holds if there exists a Borel subset $Z \subseteq(2)^{G}$ with $\mu(Z)=1$ such that $E_{G}\left\lceil Z \leq_{B} E_{H}^{Y}\right.$. Theorem 2.2 .2 is then an immediate corollary of the following: 
Theorem 2.3.5. If $E$ is an essentially free countable Borel equivalence relation, then there exists a countable group $G$ such that $E_{G} \not \mathbb{Z}_{B} E$.

Proof. We can suppose that $E=E_{H}^{X}$ is realized by a free Borel action on $X$ of the countable group $H$. Let $L$ be a finitely generated group which does not embed into $H$. Let $S=L * \mathbb{Z}$ and let $G=S L_{3}(\mathbb{Z}) \times S$. Then $G$ has no finite normal subgroups and so there does not exist a virtual embedding $\pi: G \rightarrow H$. It follows that $E_{G} \not_{B} E_{H}^{X}$.

2.4. Free and Non-Essentially Free Countable Borel Equivalence Relations. We now use 2.3.4 to show that there are continuum many free countable Borel equivalence relations. For each prime $p \in \mathbb{P}$, let $A_{p}=\bigoplus_{i=0}^{\infty} C_{p}$, and for each subset $C \subseteq \mathbb{P}$, let

$$
G_{C}=S L_{3}(\mathbb{Z}) \times \bigoplus_{p \in C} A_{p}
$$

The desired result is then an immediate consequence of the following:

Theorem 2.4.1. If $C, D \subseteq \mathbb{P}$, then $E_{G_{C}} \leq_{B} E_{G_{D}}$ iff $C \subseteq D$.

Proof. If $C \subseteq D$, then $G_{C} \leq G_{D}$, and hence $E_{G_{C}} \leq_{B} E_{G_{D}}$. Conversely, applying 2.3.4, if $E_{G_{C}} \leq_{B} E_{G_{D}}$, then there exists a virtual embedding $\pi: G_{C} \rightarrow G_{D}$. Since $S L_{3}(\mathbb{Z})$ contains a torsion-free subgroup of finite index, it follows that for each $p \in C$, the cyclic group $C_{p}$ embeds into $\bigoplus_{q \in D} A_{q}$. This implies that $p \in D$.

We now show that there also exist continuum many non-essentially free countable Borel equivalence relations. We begin by introducing the notion of ergodicity.

Definition 2.4.2. Let $G$ be a countable group and let $X$ be a standard Borel $G$-space with invariant probability measure $\mu$. Then the action of $G$ on $(X, \mu)$ is said to be ergodic iff $\mu(A)=0$ or $\mu(A)=1$ for every $G$-invariant Borel subset $A \subseteq X$.

For example, every countable group $G$ acts ergodically on $\left((2)^{G}, \mu\right)$. The following characterization of ergodicity is well known.

Theorem 2.4.3. If $\mu$ is a $G$-invariant probability measure on the standard Borel $G$-space $X$, then the following statements are equivalent.

- The action of $G$ on $(X, \mu)$ is ergodic.

- If $Y$ is a standard Borel space and $f: X \rightarrow Y$ is a G-invariant Borel function, then there exists a $G$-invariant Borel subset $M \subseteq X$ with $\mu(M)=1$ such that $f \uparrow M$ is a constant function. 
Next we need another definition.

Definition 2.4.4. The countable groups $G, H$ are called virtually isomorphic iff there exist finite normal subgroups $N \triangleleft G, M \triangleleft H$ such that $G / N \cong H / M$.

The groups defined in the following lemma will be used to construct below the desired examples of non-essentially free countable Borel equivalence relations.

Lemma 2.4.5. There exists a Borel family $\left\{S_{x} \mid x \in 2^{\mathbb{N}}\right\}$ of finitely generated groups such that if $G_{x}=S L_{3}(\mathbb{Z}) \times S_{x}$, then the following conditions hold:

- If $x \neq y$, then $G_{x}$ and $G_{y}$ are not virtually isomorphic.

- If $x \neq y$, then $G_{x}$ does not virtually embed in $G_{y}$.

Now, for each Borel subset $A \subseteq 2^{\mathbb{N}}$, let $E_{A}=\bigsqcup_{x \in A} E_{G_{x}}$.

Lemma 2.4.6. If the Borel subset $A \subseteq 2^{\mathbb{N}}$ is uncountable, then $E_{A}$ is not essentially free.

Proof. Suppose that $E_{A} \leq_{B} E_{H}^{Y}$, where $H$ is a countable group and $Y$ is a free standard Borel $H$-space. Then for each $x \in A$, we have that $E_{G_{x}} \leq_{B} E_{H}^{Y}$ and so there exists a virtual embedding $\pi_{x}: G_{x} \rightarrow H$. Since $A$ is uncountable and each $G_{x}$ is finitely generated, there exist $x \neq y \in A$ such that $\pi_{x}\left[G_{x}\right]=\pi_{y}\left[G_{y}\right]$. But then $G_{x}, G_{y}$ are virtually isomorphic, which is a contradiction.

Lemma 2.4.7. $E_{A} \leq_{B} E_{B}$ iff $A \subseteq B$.

Proof. Suppose that $E_{A} \leq_{B} E_{B}$. Suppose also that $A \nsubseteq B$ and that $x \in A \backslash B$. Then there exists a Borel reduction

$$
f:(2)^{G_{x}} \rightarrow \bigsqcup_{y \in B}(2)^{G_{y}}
$$

from $E_{G_{x}}$ to $E_{B}$. By ergodicity, there exists a $\mu_{x}$-measure 1 subset of $(2)^{G_{x}}$ which maps to a fixed $(2)^{G_{y}}$. This yields a $\mu_{x}$-nontrivial Borel homomorphism from $E_{G_{x}}$ to $E_{G_{y}}$ and so $G_{x}$ virtually embeds into $G_{y}$, which is a contradiction.

The existence of uncountably many non-essentially free countably Borel equivalence relations is an immediate consequence of Lemmas 2.4.6 and 2.4.7. 


\section{THIRD SESSION}

3.1. Ergodicity, Strong Mixing, and Borel Cocycles. In this section, we introduce some of the background theory necessary to understand the statement of Popa's Cocycle Superrigidity Theorem and the proof of Theorem 2.3.4. As usual, if a countable group $G$ acts on a standard probability space $(X, \mu)$, then we assume the action to be both free and measure-preserving so that we may stand some chance of recovering the group $G$ and its action on $X$ from the orbit equivalence relation $E_{G}^{X}$.

Recall now that the measure-preserving action of a countable group $G$ on a standard Borel probability $G$-space $(X, \mu)$ is ergodic iff every $G$-invariant subset of $X$ is null or conull. Recall also that if $\mu$ is a $G$-invariant probability measure on the standard Borel $G$-space $X$, then the action of $G$ on $(X, \mu)$ is ergodic if and only if every $G$-invariant Borel function $f: X \rightarrow Y$ into a standard Borel space $Y$ is constant on an invariant Borel set $M \subseteq X$ with $\mu(M)=1$. Thus ergodicity is a natural obstruction to smoothness: if $(X, \mu)$ is a standard Borel $G$-space where $G$ acts ergodically and preserves the nonatomic probability measure $\mu$, then $E_{G}^{X}$ is not smooth.

Definition 3.1.1. The action of $G$ on the standard probability space $(X, \mu)$ is strongly mixing iff for any Borel subsets $A, B \subseteq X$, we have that

$$
\mu(g(A) \cap B) \rightarrow \mu(A) \cdot \mu(B) \text { as } g \rightarrow \infty .
$$

In other words, if $\left\langle g_{n} \mid n \in \mathbb{N}\right\rangle$ is any sequence of distinct elements of $G$, then

$$
\lim _{n \rightarrow \infty} \mu\left(g_{n}(A) \cap B\right)=\mu(A) \cdot \mu(B) .
$$

Mixing may be viewed as a strong form of ergodicity. Indeed, suppose that the action of $G$ on $(X, \mu)$ is strongly mixing, and let $A \subseteq X$ be a $G$-invariant Borel subset. Then

$$
\mu(A)^{2}=\lim _{g \rightarrow \infty} \mu(g(A) \cap A)=\lim _{g \rightarrow \infty} \mu(A)=\mu(A),
$$

which implies that $\mu(A)=0$ or 1 . Hence strongly mixing actions are ergodic. Unlike ergodicity, however, strong mixing is a property that passes to infinite subgroups.

Observation 3.1.2. If the action of $G$ on $(X, \mu)$ is strongly mixing and $H \leq G$ is an infinite subgroup of $G$, then the action of $H$ on $(X, \mu)$ is also strongly mixing.

That the above observations actually apply to our setting is given by the following: 
Theorem 3.1.3. The action of $G$ on $\left((2)^{G}, \mu\right)$ is strongly mixing.

Proof. Consider the case when there exist finite subsets $S, T \subseteq G$ and subsets $\mathcal{F} \subseteq 2^{S}, \mathcal{G} \subseteq 2^{T}$ such that $A=\left\{f \in(2)^{G} \mid f \uparrow S \in \mathcal{F}\right\}$ and $B=\left\{f \in(2)^{G} \mid f \uparrow T \in \mathcal{G}\right\}$. If $\left\langle g_{n} \mid n \in \mathbb{N}\right\rangle$ is a sequence of distinct elements of $G$, then $g_{n}(S) \cap T=\emptyset$ for all but finitely many $n$. This means that $g_{n}(A)$ and $B$ are independent events, and so, as desired,

$$
\mu\left(g_{n}(A) \cap B\right)=\mu\left(g_{n}(A)\right) \cdot \mu(B)=\mu(A) \cdot \mu(B) .
$$

The last remaining important concept which we must introduce before stating Popa's Theorem is that of a Borel cocycle. Let $G$ and $H$ be countable discrete groups, $X$ a standard Borel $G$-space with invariant Borel probability measure $\mu$. A Borel map $\alpha: G \times X \rightarrow H$ is a cocycle iff $\alpha$ satisfies the cocycle identity

$$
\forall g, h \in G \quad \alpha(h g, x)=\alpha(h, g x) \alpha(g, x) \quad \mu \text {-a.e. }(x) \text {. }
$$

If $\beta: G \times X \rightarrow H$ is another cocyle into $H$, then we say that $\alpha$ and $\beta$ are equivalent, and write $\alpha \sim \beta$, iff there is a Borel map $b: X \rightarrow H$ such that

$$
\forall g \in G \quad \beta(g, x)=b(g x) \alpha(g, x) b(x)^{-1} \quad \mu \text {-a.e. }(x) .
$$

It is clear that $\sim$ is an equivalence relation on cocycles $G \times X \rightarrow H$.

For our purposes cocycles $\alpha: G \times X \rightarrow H$ shall always arise from Borel homomorphisms into free standard Borel $H$-spaces in the following way: suppose that $Y$ is a free standard Borel $H$-space and that $f$ is a Borel homomorphism from $E_{G}^{X}$ to $E_{H}^{Y}$. Then the map $\alpha: G \times X \rightarrow H$ defined by

$$
\alpha(g, x) f(x)=f(g x)
$$

is a cocycle. Moreoever, if $\alpha$ is the cocycle corresponding in this manner to the Borel homomorphism $f: X \rightarrow Y$, and if $b: X \rightarrow H$ is any Borel function, then the map $f^{\prime}: X \rightarrow Y$ defined by $f^{\prime}(x)=b(x) f(x)$ is also a Borel homomorphism, and the corresponding cocycle $\beta$ is equivalent to $\alpha$ via the the equation

$$
\beta(g, x)=b(g x) \alpha(g, x) b(x)^{-1}
$$

Equivalence of cocycles can be easily visualized with the aid of the following diagram: 


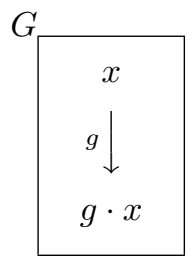

$(X, \mu)$

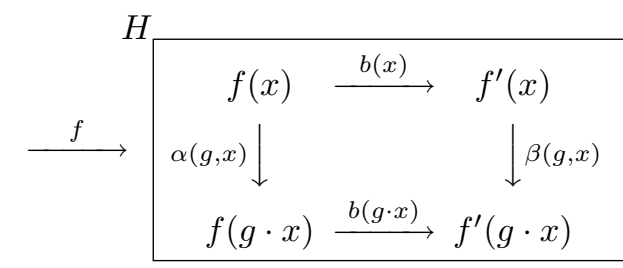

$Y$

Notice that if a cocycle $\alpha: G \times X \rightarrow H$ can be written as a function of only one variable, in the form $\alpha(g, x)=\alpha(g)$, then $\alpha$ is in fact a group homomorphism from $G$ to $H$; and the corresponding Borel homomorphism $f: X \rightarrow Y$ is, together with $\alpha$, a permutation group homomorphism $(G, X) \rightarrow(H, Y)$.

3.2. Popa's Cocycle Superrigidity Theorem and the Proof of Theorem 2.3.4. We are now ready to state (a special case of) Popa's Cocycle Superrigidity Theorem [23].

Theorem 3.2.1 (Popa). Let $\Gamma$ be a countably infinite Kazhdan group and let $G$ be a countable group such that $\Gamma \triangleleft G$. If $H$ is any countable group, then every Borel cocycle

$$
\alpha: G \times(2)^{G} \rightarrow H
$$

is equivalent to a group homomorphism of $G$ into $H$.

For example, we may let $\Gamma=S L_{n}(\mathbb{Z})$ for any $n \geq 3$, and $G=\Gamma \times S$, where $S$ is any countable group. We are now ready to prove Theorem 2.3.4, which we restate now for convenience. Recall that $E_{G}$ denotes the orbit equivalence relation of the Bernoulli action of the countable group $G$ on $\left((2)^{G}, \mu\right)$. Suppose $G=S L_{3}(\mathbb{Z}) \times S$ and that $Y$ is a free standard Borel $H$-space, where $S$ and $H$ are any countable groups. Then Theorem 2.3.4 states that if there exists a $\mu$-nontrivial Borel homomorphism from $E_{G}$ to $E_{H}^{Y}$, then there exists a virtual embedding $\pi: G \rightarrow H$.

Proof of Theorem 2.3.4. Suppose the $f:(2)^{G} \rightarrow Y$ is a $\mu$-nontrivial Borel homomorphism from $E_{G}$ to $E_{H}^{Y}$. Then we can define a Borel cocycle $\alpha: G \times(2)^{G} \rightarrow H$ by

$$
\alpha(g, x)=\text { the unique } h \in H \text { such that } h \cdot f(x)=f(g \cdot x) .
$$

By 3.2.1, after deleting a null set and adjusting $f$ if necessary, we can suppose that $\alpha: G \rightarrow H$ is a group homomorphism. 
Now, suppose that $K=\operatorname{ker} \alpha$ is infinite. Note that if $k \in K$, then $f(k \cdot x)=\alpha(k) \cdot x=f(x)$, and so $f:(2)^{G} \rightarrow X$ is $K$-invariant. Also since the action of $G$ is strongly mixing, it follows that $K$ acts ergodically on $\left((2)^{G}, \mu\right)$. But then the $K$-invariant function $f:(2)^{G} \rightarrow X$ is $\mu$-a.e. constant, which is a contradiction.

3.3. Torsion-free Abelian Groups of Finite Rank. Recall that an additive subgroup $G \leq \mathbb{Q}^{n}$ has rank $n$ iff $G$ contains $n$ linearly independent elements, and that we previously defined the standard Borel space $R\left(\mathbb{Q}^{n}\right)$ of torsion-free abelian groups of rank $n$ to be

$$
R\left(\mathbb{Q}^{n}\right)=\left\{A \leq \mathbb{Q}^{n} \mid A \text { contains a basis of } \mathbb{Q}^{n}\right\} .
$$

Recall also that for $A, B \in R\left(\mathbb{Q}^{n}\right)$, we have

$$
A \cong B \quad \text { iff } \quad \text { there exists } g \in G L_{n}(\mathbb{Q}) \text { such that } g(A)=B
$$

Thus the isomorphism relation $\cong_{n}$ on $R\left(\mathbb{Q}^{n}\right)$ is the orbit equivalence relation arising from the action of $G L_{n}(\mathbb{Q})$ on $R\left(\mathbb{Q}^{n}\right)$.

In 1937, Baer [4] gave a satisfactory classification of the rank 1 groups, which showed that $\cong_{1}$ is hyperfinite. In 1938, Kurosh [19] and Malcev [20] independently gave unsatisfactory classifications of the higher rank groups. In light of this failure to classify even the rank 2 groups in a satisfactory way, Hjorth and Kechris conjectured in 1996 [13] that the isomorphism relation for the torsion-free abelian groups of rank 2 is countable universal. As an initial step towards establishing this result, Hjorth then proved in 1998 [12] that the classification problem for the rank 2 groups is strictly harder than that for the rank 1 groups; that is, Hjorth proved that $\cong_{1}<_{B} \cong_{2}$. Soon afterwards, making essential use of the techniques of Hjorth [12] and Adams-Kechris [2], Thomas obtained the following [28]:

Theorem 3.3.1 (Thomas 2000). The complexity of the classification problems for the torsionfree abelian groups of rank $n$ increases strictly with the rank $n$.

Of course, this implies that none of the relations $\cong_{n}$ is countable universal. It remained open, however, whether the isomorphism relation on the space of torsion-free abelian groups of finite rank was countable universal. In 2006 [27], Thomas was able to show that it is not.

Theorem 3.3.2 (Thomas 2006). The isomorphism relation on the space of torsion-free abelian groups of finite rank is not countable universal. 
In the next couple of sections, we shall present an outline of the proof of 3.3.2. We begin with the notion of $E_{0}$-ergodicity, which shall play an important role at the end of the proof.

3.4. $E_{0}$-ergodicity. The following is a useful generalization of ergodicity.

Definition 3.4.1. Let $E, F$ be countable Borel equivalence relations on $X, Y$ and let $\mu$ be an E-invariant probability measure on $X$. Then we say that $E$ is F-ergodic iff every Borel homomorphism $f: X \rightarrow Y$ from $E$ to $F$ is $\mu$-trivial.

Thus $\operatorname{id}_{\mathbb{R}}$-ergodicity coincides with the usual ergodicity. Furthermore, observe that if $E$ is $F$-ergodic and $F^{\prime} \leq_{B} F$, then $E$ is also $F^{\prime}$-ergodic. We now introduce a characterization of $E_{0}$-ergodicity due to Jones and Schmidt [16].

Definition 3.4.2. Let $E=E_{G}^{X}$ be a countable Borel equivalence relation and let $\mu$ be an E-invariant probability measure on $X$. Then $E$ has nontrivial almost invariant subsets iff there exists a sequence of Borel subsets $\left\langle A_{n} \subseteq X \mid n \in \mathbb{N}\right\rangle$ satisfying the following conditions:

- $\mu\left(g \cdot A_{n} \triangle A_{n}\right) \rightarrow 0$ for all $g \in G$.

- There exists $\delta>0$ such that $\delta<\mu\left(A_{n}\right)<1-\delta$ for all $n \in \mathbb{N}$.

Theorem 3.4.3 (Jones-Schmidt). $E$ is $E_{0}$-ergodic iff $E$ has no nontrivial almost invariant subsets.

This can in turn be used to prove the following:

Theorem 3.4.4 (Jones-Schmidt). Let $G$ be a countable group and let $H \leq G$ be a nonamenable subgroup. then the shift action of $H$ on $\left((2)^{G}, \mu\right)$ is $E_{0}$-ergodic.

Finally, we remark for later use that if $E$ is $E_{0}$-ergodic and $F$ is hyperfinite, then $E$ is $F$-ergodic. We are now ready to commence with a sketch of the proof of the non-universality of the isomorphism relation on the space of torsion-free abelian groups of finite rank.

\subsection{The Non-universality of the Isomorphism Relation on Torsion-free Abelian} Groups of Finite Rank. Roughly speaking, our strategy of proof will be as follows. We know that a smooth disjoint union of countably many essentially free countable Borel equivalence relations is itself essentially free, and we know that the class of essentially free countable Borel equivalence relations does not admit a universal element. Since the isomorphism relation on the space of torsion-free abelian groups of finite rank is the smooth disjoint union of the 
$\cong_{n}$ relations, $n \geq 1$, it would suffice to show that each $\cong_{n}$ is essentially free. Unfortunately, it appears to be difficult to determine whether this is even true for $n \geq 2$. However, we shall see that coarsening each $\cong_{n}$ by the hyperfinite relation of quasi-equality yields a relation that is essentially free. In this way each $\cong_{n}$ is seen to be "(hyperfinite)-by-(essentially free)," and this will suffice to prove the non-universality of the isomorphism relation. We now proceed with the details.

Let $G=S L_{3}(\mathbb{Z}) \times S$, where $S$ is a suitably chosen countable group that we shall describe at a later stage in the proof. Let $E=E_{G}$ be the orbit equivalence relation arising from the action of $G$ on $\left((2)^{G}, \mu\right)$.

Suppose that

$$
f:(2)^{G} \rightarrow \bigsqcup_{n \geq 1} R\left(\mathbb{Q}^{n}\right)
$$

is a Borel reduction from $E$ to the isomorphism relation. After deleting a null set of $(2)^{G}$ if necessary, we may assume that $f$ takes values in $R\left(\mathbb{Q}^{n}\right)$ for some fixed $n \geq 1$.

At this point we would like to define a Borel cocycle corresponding to $f$, but unfortunately $G L_{n}(\mathbb{Q})$ does not act freely on $R\left(\mathbb{Q}^{n}\right)$. In fact, the stabilizer of each $B \in R\left(\mathbb{Q}^{n}\right)$ under the action of $G L_{n}(\mathbb{Q})$ is precisely its automorphism group $\operatorname{Aut}(B)$. We shall overcome this difficulty by shifting our focus from the isomorphism relation on $R\left(\mathbb{Q}^{n}\right)$ to the coarser quasiisomorphism relation.

Definition 3.5.1. If $A, B \in R\left(\mathbb{Q}^{n}\right)$, then $A$ and $B$ are said to be quasi-equal, written $A \approx_{n} B$, iff $A \cap B$ has finite index in both $A$ and $B$.

Theorem 3.5.2 (Thomas [28]). The quasi-equality relation $\approx_{n}$ is hyperfinite.

For each $A \in R\left(\mathbb{Q}^{n}\right)$, let $[A]$ be the $\approx_{n}$-class containing $A$. We shall consider the induced action of $G L_{n}(\mathbb{Q})$ on the set $X=\left\{[A] \mid A \in R\left(\mathbb{Q}^{n}\right)\right\}$ of $\approx_{n}$-classes. Of course, since $\approx_{n}$ is not smooth, $X$ is not a standard Borel space; but fortunately this will not pose a problem in what follows. In order to describe the setwise stabilizer in $G L_{n}(\mathbb{Q})$ of each $\approx_{n}$-class $[A]$, we now make some new definitions.

Definition 3.5.3. For each $A \in R\left(\mathbb{Q}^{n}\right)$, the ring of quasi-endomorphisms is

$$
Q E(A)=\left\{\varphi \in \operatorname{Mat}_{n}(\mathbb{Q}) \mid(\exists m \geq 1) m_{\varphi} \in \operatorname{End}(A)\right\} .
$$

Clearly $\mathrm{QE}(A)$ is a $\mathbb{Q}$-subalgebra of $\operatorname{Mat}_{n}(\mathbb{Q})$, and so there are only countably many possibilities for $\mathrm{QE}(A)$, a fact which will be of crucial importance below. 
Definition 3.5.4. $\mathrm{QAut}(A)$ is the group of units of the $\mathbb{Q}$-algebra $\mathrm{QE}(A)$.

Lemma 3.5.5. If $A \in R\left(\mathbb{Q}^{n}\right)$, then $\operatorname{QAut}(A)$ is the setwise stabilizer of $[A]$ in $G L_{n}(\mathbb{Q})$.

For each $x \in(2)^{G}$, let $A_{x}=f(x) \in R\left(\mathbb{Q}^{n}\right)$. Since there are only countably many possibilities for the group QAut $\left(A_{x}\right)$, there exists a fixed subgroup $L \leq G L_{n}(\mathbb{Q})$ and a Borel subset $X \subseteq(2)^{G}$ with $\mu(X)>0$ such that $\operatorname{QAut}\left(A_{x}\right)=L$ for all $x \in X$. Since $G$ acts ergodically on $\left((2)^{G}, \mu\right)$, it follows that $\mu(G \cdot X)=1$. In order to simplify notation, we shall assume that $G \cdot X=(2)^{G}$. After slightly adjusting $f$ if necessary, we can suppose that $\operatorname{QAut}\left(A_{x}\right)=L$ for all $x \in(2)^{G}$.

Note that the quotient group $H=N_{G L_{n}(\mathbb{Q})}(L) / L$ acts freely on the corresponding set $Y=\{[A] \mid \operatorname{QAut}(A)=L\}$ of $\approx_{n}$-classes. Hence we can define a corresponding cocycle

$$
\alpha: G \times(2)^{G} \rightarrow H
$$

by setting

$$
\alpha(g, x)=\text { the unique } h \in H \text { such that } h \cdot\left[A_{x}\right]=\left[A_{g \cdot x}\right] \text {. }
$$

Now let $S$ be a countable simple nonamenable group which does not embed into any of the countably many possibilities for $H$. Applying Theorem 3.2.1, after deleting a null set and slightly adjusting $f$ if necessary, we can suppose that

$$
\alpha: G=S L_{3}(\mathbb{Z}) \times S \rightarrow H
$$

is a group homomorphism. Since $S \leq \operatorname{ker} \alpha$, it follows that $f:(2)^{G} \rightarrow R\left(\mathbb{Q}^{n}\right)$ is a Borel homomorphism from the $S$-action on $(2)^{G}$ to the hyperfinite quasi-equality $\approx_{n}$-relation. Since $S$ is nonamenable, the $S$-action on $(2)^{G}$ is $E_{0}$-ergodic and hence $\mu$-almost all $x \in(2)^{G}$ are mapped to a single $\approx_{n}$-class, which is a contradiction. This completes the proof of Theorem 3.3.2.

\section{Fourth SEssion}

4.1. Containment vs. Borel Reducibility. Our next goal will be to present some applications of Ioana's Cocycle Superrigidity Theorem. We shall focus on a problem that was initially raised in the context of Kechris' Conjecture that $\equiv_{T}$ is universal. Recall that $\equiv_{T}$ denotes the Turing equivalence relation on $\mathcal{P}(\mathbb{N})$, so that $A \equiv_{T} B$ iff $A$ and $B$ are Turing reducible to each other. Recall also that the translation action of the free group $\mathbb{F}_{2}$ on its power set gives rise to a universal countable Borel equivalence relation, denoted by $E_{\infty}$. Now, 
if we identify $\mathbb{F}_{2}$ with a suitably chosen group of recursive permutations of $\mathbb{N}$, we see that $E_{\infty}$ may be realized as a subset of $\equiv_{T}$. Thus the following conjecture of Hjorth [3] would imply that $\equiv_{T}$ is universal.

Conjecture 4.1.1 (Hjorth). If $F$ is a universal countable Borel equivalence relation on the standard Borel space $X$ and $E$ is a countable Borel equivalence relation such that $F \subseteq E$, then $E$ is also universal.

In [28], Thomas pointed out that it was not even known whether there existed a pair $F \subseteq E$ of countable Borel equivalence relations for which $F \mathbb{Z}_{B} E$. Soon afterwards, Adams [1] constructed a pair of countable Borel equivalence relations $F \subseteq E$ which were incomparable with respect to Borel reducibility. In the remainder of this session, as an application of Ioana Superrigidity, we shall sketch a proof of the following:

Theorem 4.1.2 (Thomas 2002). There exists a pair of countable Borel equivalence relations $F \subseteq E$ on a standard Borel space $X$ such that $E<_{B} F$.

Here $E$ and $F$ will arise from the actions of $S L_{n}(\mathbb{Z})$ and a suitable congruence subgroup on $S L_{n}\left(\mathbb{Z}_{p}\right)$. We shall first need to recall some basic facts about $\mathbb{Z}_{p}$.

\subsection{The Ring $\mathbb{Z}_{p}$ of $p$-adic Integers.}

Definition 4.2.1. The ring $\mathbb{Z}_{p}$ of p-adic integers is the inverse limit of the system

$$
\cdots \stackrel{\varphi_{n+1}}{\longrightarrow} \mathbb{Z} / p^{n+1} \mathbb{Z} \stackrel{\varphi_{n}}{\longrightarrow} \mathbb{Z} / p^{n} \mathbb{Z} \stackrel{\varphi_{n-1}}{\longrightarrow} \cdots \stackrel{\varphi_{1}}{\longrightarrow} \mathbb{Z} / p \mathbb{Z} .
$$

It is useful to think of $p$-adic integers as formal sums

$$
z=a_{0}+a_{1} p+a_{2} p^{2}+\cdots+a_{n} p^{n}+\cdots
$$

where each $0 \leq a_{n}<p$. We define the $p$-adic norm ||$_{p}$ by

$$
|z|_{p}=p^{-\operatorname{ord}_{p}(z)}, \quad \operatorname{ord}_{p}(z)=\min \left\{n \mid a_{n} \neq 0\right\},
$$

and the $p$-adic metric by

$$
d_{p}(x, y)=|x-y|_{p} .
$$

With this metric, $\mathbb{Z}_{p}$ is a compact Polish space having the integers as a dense subring. It follows that $S L_{n}\left(\mathbb{Z}_{p}\right)$ is a compact Polish group with dense subgroup $S L_{n}(\mathbb{Z}) \leq S L_{n}\left(\mathbb{Z}_{p}\right)$. Note that $S L_{n}\left(\mathbb{Z}_{p}\right)$ is the inverse limit of the system

$$
\cdots \stackrel{\theta_{n+1}}{\longrightarrow} S L_{n}\left(\mathbb{Z} / p^{n+1} \mathbb{Z}\right) \stackrel{\theta_{n}}{\longrightarrow} S L_{n}\left(\mathbb{Z} / p^{n} \mathbb{Z}\right) \stackrel{\theta_{n-1}}{\longrightarrow} \cdots \stackrel{\theta_{1}}{\longrightarrow} S L_{n}(\mathbb{Z} / p \mathbb{Z}) .
$$


Since $S L_{n}\left(\mathbb{Z}_{p}\right)$ is compact, there exists a unique Haar probability measure on $S L_{n}\left(\mathbb{Z}_{p}\right)$ (ie, a unique a probability measure $\mu_{p}$ which is invariant under the left translation action). In fact, $\mu_{p}$ is simply the inverse limit of the counting measures on

$$
\cdots \stackrel{\theta_{n+1}}{\longrightarrow} S L_{n}\left(\mathbb{Z} / p^{n+1} \mathbb{Z}\right) \stackrel{\theta_{n}}{\longrightarrow} S L_{n}\left(\mathbb{Z} / p^{n} \mathbb{Z}\right) \stackrel{\theta_{n-1}}{\longrightarrow} \cdots \stackrel{\theta_{1}}{\longrightarrow} S L_{n}(\mathbb{Z} / p \mathbb{Z}) .
$$

Observe that if $H \leq S L_{n}\left(\mathbb{Z}_{p}\right)$ is an open subgroup, then $H$ has finite index in $S L_{n}\left(\mathbb{Z}_{p}\right)$ and

$$
\mu_{p}(H)=\frac{1}{\left[S L_{n}\left(\mathbb{Z}_{p}\right): H\right]} .
$$

We claim further that there are no other $S L_{n}(\mathbb{Z})$-invariant probability measures on $S L_{n}\left(\mathbb{Z}_{p}\right)$.

Theorem 4.2.2. $\mu_{p}$ is the unique $S L_{n}(\mathbb{Z})$-invariant probability measure on $S L_{n}\left(\mathbb{Z}_{p}\right)$.

Proof. First note that $S L_{n}(\mathbb{Z})$ acts continuously on the space $\mathcal{M}$ of probability measures on $S L_{n}\left(\mathbb{Z}_{p}\right)$. Hence if $\nu$ is any probability measure on $S L_{n}\left(\mathbb{Z}_{p}\right)$, then

$$
S_{\nu}=\left\{g \in S L_{n}\left(\mathbb{Z}_{p}\right) \mid \nu \text { is } g \text {-invariant }\right\}
$$

is a closed subgroup of $S L_{n}\left(\mathbb{Z}_{p}\right)$. Thus, by density, any $S L_{n}(\mathbb{Z})$-invariant probability measure is actually $S L_{n}\left(\mathbb{Z}_{p}\right)$-invariant, and so must be $\mu_{p}$.

4.3. Unique Ergodicity and Ergodic Components. We define an action of a group $G$ on a standard Borel $G$-space $X$ to be uniquely ergodic iff there exists a unique $G$-invaraint probability measure $\mu$ on $X$. In this case, it is well known that $\mu$ must be ergodic. For if there were a $G$-invariant Borel set $A \subseteq X$ with $0<\mu(A)<1$, then we could define distinct $G$-invariant probability measures by

$$
\begin{aligned}
& \nu_{1}(Z)=\mu(Z \cap A) / \mu(A) \\
& \nu_{2}(Z)=\mu(Z \backslash A) / \mu(X \backslash A) .
\end{aligned}
$$

Note that 4.2 .2 above simply states that the action of $S L_{n}(\mathbb{Z})$ on $S L_{n}\left(\mathbb{Z}_{p}\right)$ is uniquely ergodic.

Next, suppose that $\Gamma$ is a countable group with finite-index subgroup $\Lambda \leq \Gamma$, and that $X$ is a standard Borel $\Gamma$-space with an invariant ergodic probability measure $\mu$. Then we call a positive measure, $\Lambda$-invariant Borel set $Z \subseteq X$ an ergodic component for the action of $\Lambda$ on $X$ iff $\Lambda$ acts ergodically on $\left(Z, \mu_{Z}\right)$, where $\mu_{Z}$ is the normalized probability measure on $Z$ defined by $\mu_{Z}(A)=\mu(A) / \mu(Z)$. It is easily checked that there exists a partition $Z_{1} \sqcup \cdots \sqcup Z_{d}$ of $X$ into finitely many ergodic components and that the collection of ergodic components is uniquely determined up to $\mu$-null sets. Furthermore, if the action of $\Gamma$ on $X$ is uniquely ergodic, then the action of $\Lambda$ on each ergodic component is also uniquely ergodic. 
Now fix a prime $p$ and for $n \geq 3$, let $S L_{n}(\mathbb{Z})$ act by translations as a subgroup on $S L_{n}\left(\mathbb{Z}_{p}\right)$. Notice that this action is ergodic but not strongly mixing. Let $\Lambda=\operatorname{ker} \varphi$ and $H=\operatorname{ker} \psi$ be the kernels of the homomorphisms

$$
\varphi: S L_{n}(\mathbb{Z}) \rightarrow S L_{n}(\mathbb{Z} / p \mathbb{Z})
$$

and

$$
\psi: S L_{n}\left(\mathbb{Z}_{p}\right) \rightarrow S L_{n}\left(\mathbb{Z}_{p} / p \mathbb{Z}_{p}\right) \cong S L_{n}(\mathbb{Z} / p \mathbb{Z}) .
$$

Then $H$ is the closure of $\Lambda$ in $S L_{n}\left(\mathbb{Z}_{p}\right)$, and the ergodic decomposition of the $\Lambda$-action is given by

$$
S L_{n}\left(\mathbb{Z}_{p}\right)=H g_{1} \sqcup \cdots \sqcup H g_{d}, \quad d=\left|S L_{n}(\mathbb{Z} / p \mathbb{Z})\right| .
$$

We are now ready to state Thomas' result.

Theorem 4.3.1. Let $n \geq 3$ and let $F \subseteq E$ be the orbit equivalence relations of the actions of $\Lambda$ and $S L_{n}(\mathbb{Z})$ on $S L_{n}\left(\mathbb{Z}_{p}\right)$. Then $E<_{B} F$.

We shall devote the next section to a proof of this result.

4.4. Proof of Theorem 4.3.1. First consider the ergodic decomposition of the $\Lambda$-action,

$$
S L_{n}\left(\mathbb{Z}_{p}\right)=H g_{1} \sqcup \cdots \sqcup H g_{d}, \quad d=\left|S L_{n}(\mathbb{Z} / p \mathbb{Z})\right| .
$$

From this we see that

$$
F=E_{1} \oplus \cdots \oplus E_{d}, \quad \text { where } E_{i}=F\left\lceil H g_{i} .\right.
$$

We claim that $E \sim_{B} E_{i}$ for each $i$.

To see that $E_{i} \leq_{B} E$, we check that the inclusion map $H g_{i} \rightarrow S L_{n}\left(\mathbb{Z}_{p}\right)$ is a Borel reduction. Given $x, y \in H g_{i}, x E_{i} y$ clearly implies $x E y$, since $F \subseteq E$; and conversely, if $x E y$, then there exists $\gamma \in S L_{n}(\mathbb{Z})$ such that $\gamma x=y$, whence $\emptyset \neq \gamma H g_{i} \cap H g_{i}=H \gamma g_{i} \cap H g_{i}$, and therefore $\gamma \in S L_{n}(\mathbb{Z}) \cap H=\Lambda$.

To show that $E \leq_{B} E_{i}$, we choose coset representatives $g_{k}$ so that each $g_{k} \in S L_{n}(\mathbb{Z})$. Then for each $1 \leq k \leq d$, define $h_{k}: H g_{k} \rightarrow H g_{i}$ by $h_{k}(x)=g_{i} g_{k}^{-1} x$. We claim that $h=h_{1} \cup \cdots \cup h_{d}$ is a Borel reduction from $E$ to $E_{i}$. For if $x, y \in S L_{n}\left(\mathbb{Z}_{p}\right)$, then

$$
\begin{array}{rll}
x E y & \text { iff } & h(x) E h(y) \\
& \text { iff } & h(x) E_{i} h(y),
\end{array}
$$


where this last equivalence follows because $h(x), h(y) \in H g_{i}$. This shows that $E \sim_{B} E_{i}$ for each $i$, and hence we have shown that

$$
F \sim_{B} \underbrace{E \oplus \cdots \oplus E}_{d \text { times }} .
$$

It will therefore be enough to prove the following:

Theorem 4.4.1 (Thomas 2002). If $n \geq 3$, then

$$
E<_{B} E \oplus E<_{B} \cdots<_{B} \underbrace{E \oplus \cdots \oplus E}_{d \text { times }}<_{B} \cdots
$$

Proof of Theorem 4.4.1. Let us first introduce some notation. We let $\Gamma=S L_{n}(\mathbb{Z})$ and write $(K, \mu)$ for $\left(S L_{n}\left(\mathbb{Z}_{p}\right), \mu_{p}\right)$, so that $E$ is the orbit equivalence relation arising from the $\Gamma$-action on $K$. It clearly suffices to show that if $f: K \rightarrow K$ is a Borel reduction from $E$ to $E$, then $\mu(\Gamma \cdot f(K))=1$.

Thus suppose $f: K \rightarrow K$ is a Borel reduction from $E$ to $E$. Since $\Gamma$ acts freely on $K$, we can define a Borel cocycle $\alpha: \Gamma \times K \rightarrow \Gamma$ by

$$
\alpha(g, x)=\text { the unique } h \in H \text { such that } h \cdot f(x)=f(g \cdot x) .
$$

By Ioana Superrigidity [14], there exists a subgroup $\Delta \leq \Gamma$ of finite index and an ergodic component $X \subseteq K$ for the $\Delta$-action such that $\alpha \uparrow(\Delta \times X)$ is equivalent to a group homomorphism

$$
\psi: \Delta \rightarrow S L_{n}(\mathbb{Z})
$$

After slightly adjusting $f$ if necessary, we can suppose that $\alpha \uparrow(\Delta \times X)=\psi$, and hence that

$$
\psi(g) \cdot f(x)=f(g \cdot x) \text { for all } g \in \Delta \text { and } x \in X \text {. }
$$

Furthermore, by Margulis Superrigidity [21], if $\Delta \leq S L_{n}(\mathbb{Z})$ is a subgroup of finite index and $\psi: \Delta \rightarrow S L_{n}(\mathbb{Z})$ is a group homomorphism, then either $\psi(\Delta)$ is finite, or $\psi$ is an embedding and $\psi(\Delta)$ is a subgroup of finite index in $S L_{n}(\mathbb{Z})$. We shall now handle each of these cases in turn.

If $\psi(\Delta)$ is finite, then we can define a $\Delta$-invariant map $\phi: X \rightarrow[K]^{<\omega}$ by

$$
\phi(x)=\{f(g \cdot x) \mid g \in \Delta\} .
$$

Since $\Delta$ acts ergodically on $X$, it follows that $\phi$ is constant on a $\mu$-conull subset of $X$, which is a contradiction. 
Supposing instead that $\psi$ is an embedding and that $\psi(\Delta)$ is a subgroup of finite index in $S L_{n}(\mathbb{Z})$, let $Y_{1}, \ldots, Y_{d}$ be the ergodic components for the action of $\psi(\Delta)$ on $K$. Since $\Delta$ acts ergodically on $X$, we can suppose that there exists $Y=Y_{i}$ such that $f: X \rightarrow Y$. Recalling that $\psi(g) \cdot f(x)=f(g \cdot x)$, we can then define a $\psi(\Delta)$-invariant probability measure $\nu$ on $Y$ by

$$
\nu(Z)=\mu\left(f^{-1}(Z)\right) / \mu(X) .
$$

As the action of $\psi(\Delta)$ on $Y$ is uniquely ergodic, $\nu(Z)=\mu(Z) / \mu(Y)$. Hence $\mu(f(X))=$ $\mu(Y)>0$, and so $\mu(\Gamma \cdot f(K))=1$, as desired. This completes the proof of 4.4.1, and hence also that of 4.3.1.

\subsection{Profinite Actions and Ioana Superrigidity.}

Definition 4.5.1. Let $\Gamma$ be a countable group. For each $n \in \mathbb{N}$, let $\left(X_{n}, \mu_{n}\right)$ be a finite $\Gamma$-space, where for $Y \subseteq X_{n}, \mu_{n}(Y)=|Y| /\left|X_{n}\right|$. Suppose that each $\left(X_{n}, \mu_{n}\right)$ is a quotient of $\left(X_{n+1}, \mu_{n+1}\right)$. Then the canonical action of $\Gamma$ on

$$
(X, \mu)=\lim _{\leftarrow}\left(X_{n}, \mu_{n}\right)
$$

is said to be a profinite action.

For example, if $K$ is a profinite group and $\Gamma, L \leq K$ are subgroups such that $\Gamma$ is countable dense and $L$ is closed, then the action of $\Gamma$ on $K / L$ is profinite. In particular, if $\Gamma$ is a residually finite group with decreasing sequence of finite index normal subgroups

$$
\Gamma=\Gamma_{0}>\Gamma_{1}>\cdots>\Gamma_{n}>\cdots
$$

such that $\bigcap \Gamma_{n}=1$, then $\Gamma$ is a dense subgoup of the profinite group $\lim \Gamma / \Gamma_{n}$, and its action as a subgroup will be profinite. Of course, this example covers the situation discussed above, ie, the action of $S L_{n}(\mathbb{Z})$ on $S L_{n}\left(\mathbb{Z}_{p}\right)$. As one further example, we remark that the action of $S L_{n}(\mathbb{Z})$ on the projective space $P G\left(n-1, \mathbb{Q}_{p}\right)$ is profinite.

We may now finally state Ioana's Cocycle Superrigidity Theorem [14], which was used above in our proof of 4.3.1.

Theorem 4.5.2 (Ioana). Let $\Gamma$ be a countably infinite Kazhdan group and let $(X, \mu)$ be a free ergodic profinite $\Gamma$-space. Suppose that $H$ is any countable group and that $\alpha: \Gamma \times X \rightarrow H$ is a Borel cocycle. Then there exists a subgroup $\Delta \leq \Gamma$ of finite index and an ergodic component $Y \subseteq X$ for the $\Delta$-action such that $\alpha \uparrow(\Delta \times Y)$ is equivalent to a homomorphism $\psi: \Delta \rightarrow H$. 
To conclude this section, we shall present a final application of Ioana's theorem.

Theorem 4.5.3 (Thomas 2002). Fix $n \geq 3$. Let $S$ be a nonempty set of primes and regard $S L_{n}(\mathbb{Z})$ as a subgroup of

$$
G(S)=\prod_{p \in S} S L_{n}\left(\mathbb{Z}_{p}\right)
$$

via the diagonal embedding. Let $E_{S}$ be the corresponding orbit equivalence relation. If $S \neq T$, then $E_{S}$ and $E_{T}$ are incomparable with respect to Borel reducibility.

Sketch of Proof. For simplicity, suppose that $S=\{p\}$ and $T=\{q\}$, where $p \neq q$ are distinct primes. Suppose that $f: S L_{n}\left(\mathbb{Z}_{p}\right) \rightarrow S L_{n}\left(\mathbb{Z}_{q}\right)$ is a Borel reduction from $E_{\{p\}}$ to $E_{\{q\}}$. Then applying Ioana Superrigidity and arguing as in the proof of Theorem 4.4.1 above, we see that after passing to subgroups of finite index and ergodic components if necessary,

$$
\left(S L_{n}(\mathbb{Z}), S L_{n}\left(\mathbb{Z}_{p}\right), \mu_{p}\right) \cong\left(S L_{n}(\mathbb{Z}), S L_{n}\left(\mathbb{Z}_{q}\right), \mu_{q}\right)
$$

as measure-preserving permutation groups. Hence it only remains to detect the prime in $\left(S L_{n}(\mathbb{Z}), S L_{n}\left(\mathbb{Z}_{p}\right), \mu_{p}\right)$

Towards this end, recall that $\operatorname{Aut}\left(S L_{n}(\mathbb{Z}), S L_{n}\left(\mathbb{Z}_{p}\right) \mu_{p}\right)$ consists of the measure-preserving bijections $\varphi: S L_{n}\left(\mathbb{Z}_{p}\right) \rightarrow S L_{n}\left(\mathbb{Z}_{p}\right)$ such that for all $\gamma \in S L_{n}(\mathbb{Z})$,

$$
\varphi(\gamma \cdot x)=\gamma \cdot \varphi(x) \text { for } \mu_{p} \text {-a.e. } x
$$

where as usual we identify two such maps if they agree $\mu_{p}$-a.e. Also notice that for each $g \in S L_{n}\left(\mathbb{Z}_{p}\right)$, we can define a corresponding automorphism $\varphi \in \operatorname{Aut}\left(S L_{n}(\mathbb{Z}), S L_{n}\left(\mathbb{Z}_{p}\right) \mu_{p}\right)$ by $\varphi(x)=x g$. The following proposition shows that there are no others.

Proposition 4.5.4 (Gefter-Golodets $1988[10])$. $\operatorname{Aut}\left(S L_{n}(\mathbb{Z}), S L_{n}\left(\mathbb{Z}_{p}\right), \mu_{p}\right)=S L_{n}\left(\mathbb{Z}_{p}\right)$.

Proof of 4.5.4. Suppose that $\varphi \in \operatorname{Aut}\left(S L_{n}(\mathbb{Z}), S L_{n}\left(\mathbb{Z}_{p}\right), \mu_{p}\right)$. For each $x \in S L_{n}\left(\mathbb{Z}_{p}\right)$, let $h(x) \in S L_{n}\left(\mathbb{Z}_{p}\right)$ be such that $\varphi(x)=x h(x)$. If $\gamma \in S L_{n}(\mathbb{Z})$, then

$$
\varphi(\gamma \cdot x)=\gamma \cdot \varphi(x)=\gamma \cdot x h(x)
$$

and so $h(\gamma \cdot x)=h(x)$. Since $S L_{n}(\mathbb{Z})$ acts ergodically on $\left(S L_{n}\left(\mathbb{Z}_{p}\right), \mu_{p}\right)$, there exists $g \in$ $S L_{n}\left(\mathbb{Z}_{p}\right)$ such that $h(x)=g$ for $\mu_{p}$-a.e. $x$. 
Thus we have reduced our problem to that of detecting the prime $p$ in the topological group $S L_{n}\left(\mathbb{Z}_{p}\right)$. But this is easy, since $S L_{n}\left(\mathbb{Z}_{p}\right)$ is virtually a pro- $p$ group. More precisely, if $H$ is any open subgroup of $S L_{n}\left(\mathbb{Z}_{p}\right)$, then

$$
\left[S L_{n}\left(\mathbb{Z}_{p}\right): H\right]=b p^{l}
$$

for some $l \geq 0$ and some divisor $b$ of $\left|S L_{n}(\mathbb{Z} / p \mathbb{Z})\right|$. This completes our sketch of a proof of Theorem 4.5.3.

4.6. Open Problems. In this closing section, we shall point out a number of open problems in the area of countable Borel equivalence relations, some of them being quite basic. Indeed, the field remains in a pre-classical stage of development, and many of the natural question one might ask are still completely open. We shall organize our survey by considering questions from the theories of the hyperfinite, treeable, and universal countable Borel equivalence relations, respectively.

4.6.1. Hyperfinite Relations. Recall that a countable Borel equivalence relation $E$ on a standard Borel space $X$ is said to be hyperfinite iff $E$ can be written as the union of a countable increasing sequence of finite Borel equivalence relations on $X$, where an equivalence relation is finite if each of its classes is finite. A theorem of Dougherty, Jackson, and Kechris [7] provides two additional characterizations:

Theorem 4.6.1 (Dougherty-Jackson-Kechris). If $E$ is a countable Borel equivalence relation on a standard Borel space $X$, then the following are equivalent:

- $E$ is hyperfinite.

- $E \leq_{B} E_{0}$.

- There exists a Borel action of $\mathbb{Z}$ on $X$ such that $E=E_{\mathbb{Z}}^{X}$.

In fact, every $\mathbb{Z}$-action on a standard Borel $\mathbb{Z}$-space $X$ yields a hyperfinite orbit equivalence relation. By a theorem of Gao and Jackson [9], even more is true.

Theorem 4.6.2 (Gao-Jackson). If $G$ is a countable abelian group and $X$ is a standard Borel $G$-space, then $E_{G}^{X}$ is hyperfinite.

An important question is how much further this result can be extended. By a theorem of Jackson, Kechris, and Louveau [15], if $G$ is a countable, nonamenable group, then the orbit equivalence relation $E_{G}$ arising from the free action of $G$ on $\left((2)^{G}, \mu\right)$ is not hyperfinite. However, the following remains open: 
Question 4.6.3 (Weiss [29]). Suppose that $G$ is a countable amenable group and that $X$ is a standard Borel $G$-space. Does it follow that $E_{G}^{X}$ is hyperfinite?

As a partial answer we have the following theorem of Connes, Feldman, and Weiss [5].

Theorem 4.6.4 (Connes-Feldman-Weiss). Suppose that $G$ is a countable amenable group and that $X$ is a standard Borel $G$-space. If $\mu$ is any Borel probability measure on $X$, then there exists a Borel subset $Y \subseteq X$ with $\mu(Y)=1$ such that $E \uparrow Y$ is hyperfinite.

Finally, recall that von Neumann proved that no amenable group contains a free nonabelian subgroup, and went on to conjecture that this property characterizes amenability. (While the conjecture probably goes back to von Neumann, and bears his name, its first official appearance was in Day [6]). Then in 1980 Ol'shanskii refuted the conjecture, exhibiting a periodic nonamenable group with no free nonabelian subgroup [22]. As a sort of analogue of the von Neumann Conjecture, we have the following:

Conjecture 4.6.5 (Kechris). If $E$ is a non-hyperfinite countable Borel equivalence relation, then there exists a non-hyperfinite treeable relation $F$ such that $F \leq_{B} E$.

\subsubsection{Treeable Relations.}

Definition 4.6.6. The countable Borel equivalence relation $E$ on $X$ is said to be treeble iff there is a Borel acyclic graph $(X, R)$ whose connected components are the E-classes.

For example, if the countable free group $\mathbb{F}$ acts freely on a standard Borel $\mathbb{F}$-space $X$, then the corresponding orbit equivalence relation $E_{\mathbb{F}}^{X}$ is treeable. Conversely, by a theorem of Jackson, Kechris, and Louveau [15], if $E$ is treeable then there exists a free Borel action of a countable free group $\mathbb{F}$ on a standard Borel space $Y$ such that $E \sim_{B} E_{\mathbb{F}}^{Y}$.

Every hyperfinite countable Borel equivalence relation is treeable; however, the universal countable Borel equivalence relation $E_{\infty}$ is not treeable, and there exist countable Borel equivalence relations that are treeable but not hyperfinte. Moreover, the class of treeable countable Borel equivalence relations admits a universal element.

Theorem 4.6.7 (Jackson-Kechris-Louveau [15]). Let $E_{\infty T}$ be the orbit equivalence relation arising from the free action of $\mathbb{F}_{2}$ on $(2)^{\mathbb{F}_{2}}$. Then $E_{\infty T}$ is universal for treeable countable Borel equivalence relations. 
Unfortunately, only three nonsmooth treeable countable Borel equivalence relations are currently known up to Borel bireducibility; namely: $E_{0}, E_{\infty T}$, and an intermediate one constructed by Hjorth. This leads to the following basic open question:

Question 4.6.8 (Jackson-Kechris-Louveau). Do there exist infinitely many nonsmooth treeable countable Borel equivalence relations up to Borel bireducibility?

Hjorth has shown that if $E$ is a profinite treeable relation, then $E<_{B} E_{\infty T}$. Consider, then, the following examples. Let $S$ be a nonempty set of primes and regard $S L_{2}(\mathbb{Z})$ as a subgroup of

$$
G(S)=\prod_{p \in S} S L_{2}\left(\mathbb{Z}_{p}\right)
$$

via the diagonal embedding. Letting $S L_{2}(\mathbb{Z})$ act by translation on $G(S)$, the corresponding orbit equivalence relation $E_{S}$ is a non-hyperfinite profinite treeable relation.

Conjecture 4.6.9 (Thomas). If $S \neq T$, then $E_{S}$ and $E_{T}$ are incomparable with respect to Borel reducibility.

Conjecture 4.6.10 (Thomas). If $S$ is any nonempty set of primes, then

$$
E_{S}<_{B} E_{S} \oplus E_{S}<_{B} \cdots<_{B} \underbrace{E_{S} \oplus \cdots \oplus E_{S}}_{n \text { times }}<_{B} \cdots
$$

4.6.3. Universal Relations. We conclude with some basic open questions concerning universal countable Borel equivalence relations. Already mentioned above was the following basic question of Hjorth [3]:

Conjecture 4.6.11 (Hjorth). If $E$ is a universal countable Borel equivalence relation on the standard Borel space $X$ and $F$ is a countable Borel equivalence relation such that $E \subseteq F$, then $F$ is also universal.

Of course, if true, this would imply that $\equiv_{T}$ is universal.

Question 4.6.12 (Jackson-Kechris-Louveau [15]). Suppose that $E$ is a universal countable Borel equivalence relation on the standard Borel space $X$ and that $Y \subseteq X$ is an E-invariant Borel subset. Does it follow that either $E \uparrow Y$ or $E \uparrow(X \backslash Y)$ is universal?

Finally we consider some questions concerning the notion of a minimal cover of an equivalence relation. 
Definition 4.6.13. If $E, E^{\prime}$ are countable Borel, then $E^{\prime}$ is a minimal cover of $E$ iff:

- $E<_{B} E^{\prime} ;$ and

- if the countable Borel $F$ satisfies $E \leq_{B} F \leq_{B} E^{\prime}$, then either $E \sim_{B} F$ or $F \sim_{B} E^{\prime}$.

Open Problem 4.6.14. Find an example of a nonsmooth countable Borel equivalence relation which has a minimal cover.

Open Problem 4.6.15. Find an example of a nonuniversal countable Borel equivalence relation which does not have a minimal cover.

\section{REFERENCES}

[1] S. Adams, Containment does not imply Borel reducibility, in: Set Theory: The Jahnal Conference (Ed: S. Thomas), DIMACS Series, vol. 58, American Mathematical Society, 2002, pp. 1-23.

[2] S. Adams and A. S. Keckris, Linear algebraic groups and countable Borel equivalence relations, J. Amer. Math. Soc. 13 (2000), 909-943.

[3] A. Andretta, R. Camerlo, and G. Hjorth, Conjugacy equivalence relations on subgroups, Fund. Math., 167 (2001), 189-212.

[4] R. Baer, Abelian groups without elements of finite order, Duke Math. Journal, 3 (1937), 68-122.

[5] A. Connes, J. Feldman, and B. Weiss, An amenable equivalence relation is generated by a single transformation, Ergodic Theory and Dynamical Systems, 1 (1981), 430-450.

[6] M. M. Day, Amenable semigroups, Illinois J. Math. 1 (1957), 509-544.

[7] R. Dougherty, S. Jackson, and A. S. Kechris, The structure of hyperfinite Borel equivalence relations, Trans. Amer. Math. Soc. 341 (1) (1994), 193-225.

[8] J. Feldman and C. C. Moore, Ergodic equivalence relations and Von Neumann algebras I, Trans. Amer. Math. Soc. 234 (1977), 289-324.

[9] S. Gao and S. Jackson, Countable abelian group actions and hyperfinite equivalence relations, preprint, 2007.

[10] S. L. Gefter and V. Ya. Golodets, Fundamental groups for ergodic actions and actions with unit fundamental groups, Publ. RIMS, Kyoto Univ. 24 (1988), 821-847.

[11] L. Harrington, A. S. Kechris, and A. Louveau, A Glimm-Effros dechotomy for Borel equivalence relations, J. Amer. Math. Soc. 3 (4) (1990), 903-928.

[12] G. Hjorth, Around nonclassifiability for countable torsion-free abelian groups, in Abelian groups and modules (Dublin, 1998), Trends Math., Birkhäuser, Basel (1999), 269-292.

[13] G. Hjorth and A. S. Kechris, Borel equivalence relations and classification of countable models, Annals of Pure and Applied Logic, 82 (1996), 221-272.

[14] A. Ioana, Cocycle superrigidity for profinite actions of Kazhdan groups, in progress.

[15] S. Jackson, A. S. Kechris, and A. Louveau, Countable Borel equivalence relations, J. Math. Logic, 2 (2002), 1-80. 
[16] V. F. R. Jones and K. Schmidt, Asymptotically invariant sequences and approximate finiteness, Amer. J. Math. 109 (1987), 91-114.

[17] A. S. Kechris, Classical Descriptive Set Theory, Graduate Texts in Mathematics 156 Springer-Verlag, New York, 1994.

[18] K. Kuratowski, Sur une généralisation de la notion d'homéomorphie, Fund. Math. 22 (1934), 206-220.

[19] A. G. Kurosh, Primitive torsionsfreie abelsche Gruppen vom endlichen Range, Ann. Math. 38 (1937), 175-203.

[20] A. I. Malcev, Torsion-free abelian groups of finite rank (Russian), Mat. Sbor. 4 (1938), 45-68.

[21] G. A. Margulis, Discrete Subgroups of Semisimple Lie Groups, Erg. der Math. und ihrer Grenz. 17, Springer-Verlag, 1991.

[22] A. Yu. Ol'shanskii, On the question of the existence of an invariant mean on a group (Russian), Uspekhi Mat. Nauk, 35 (1980), no. 4, 199-200.

[23] S. Popa, Cocycle and orbit equivalence superrigidity for malleable actions of $\omega$-rigid groups, Inventiones Mathematicae, 170 (2) (2007), 243-295.

[24] J. Silver, Counting the number of equivalence classes of Borel and co-analytic equivalence relations, Ann. Math. Logic, 18 (1980), 1-28.

[25] T. Slaman and J. Steel, Definable functions on degrees, in: Cabal Seminar 81-85, Lecture Notes in Mathematics, 1333, Springer-Verlag (1998), 37-55

[26] S. M. Srivastava, A Course on Borel Sets, Graduate Texts in Mathematics 180, Springer-Verlag, 1998.

[27] S. Thomas, Popa superrigidity and countable Borel equivalence relations, accepted for publication in Ann. Pure Appl. Logic as of 7/31/07.

[28] S. Thomas, The classification problem for torsion-free abelian groups of finite rank, J. Amer. Math. Soc. 16 (2003), 233-258.

[29] B. Weiss, Measureable dynamics, Conference in modern analysis and probability (R. Beals et al., editors), Contemporary Mathematics, vol. 26, American Mathematical Society, Providence, Rhode Island, 1984, 395-421. 